\title{
Near infrared photoimmunotherapy using a fiber optic diffuser for treating peritoneal gastric cancer dissemination
}

\author{
Tadanobu Nagaya $^{1} \cdot$ Shuhei Okuyama ${ }^{1} \cdot$ Fusa Ogata $^{1} \cdot$ Yasuhiro Maruoka $^{1} \cdot$ Peter L. Choyke $^{1} \cdot$ Hisataka Kobayashi $^{1} \mathbb{C}$
}

Received: 31 January 2018 / Accepted: 20 August 2018 / Published online: 31 August 2018

(c) This is a U.S. government work and its text is not subject to copyright protection in the United States; however, its text may be subject to foreign copyright protection 2018

\begin{abstract}
Background Peritoneal dissemination (PD) of abdominal malignancies is a common form of metastasis and its presence signals a poor prognosis. New treatment is required for patients with PD. Near infrared photoimmunotherapy (NIR-PIT) is a highly selective tumor treatment that employs an antibody-photo-absorber conjugate (APC). In this study, we investigate in vitro and in vivo efficacy of trastuzumab (tra)-IR700DX NIR-PIT on a human epidermal growth factor receptor type 2 (HER2)-positive gastric cancer cell line.

Methods NIR-PIT effects were investigated in vitro and in vivo. Disseminated peritoneal implants mice were separated into 5 groups: (1) no treatment; (2) tra-IR700 i.v. only; (3) NIR light only; (4) NIR-PIT; (5) repeated NIR-PIT. The peritoneal cavity was irradiated with NIR light using a fiber optic diffuser delivered through the catheter.

Results Specific binding and cell-specific killing was observed after NIR-PIT in vitro. In the in vivo study, fluorescence endoscopy showed high tumor accumulation of tra-IR700 within tumors. Significantly prolonged survival was achieved in the three treatment groups (tra-IR700 i.v. only, NIR-PIT, and repeated NIR-PIT groups) compared with control and NIR light only group ( $p<0.05$ for tra-IR700 i.v. only, $p<0.01$ for NIR-PIT, and $p<0.0001$ for repeated NIR-PIT). Moreover, most prolonged survival was shown for the repeated NIR-PIT group ( $p<0.0001$ vs tra-IR700 i.v. only, $p<0.01$ vs NIR-PIT). Conclusion NIR-PIT using a fiber optic diffuser to deliver light is a promising candidate for the treatment of disseminated peritoneal metastases and could be readily translated to humans.
\end{abstract}

Keywords Near infrared photoimmunotherapy · Gastric cancer $\cdot$ Fiber optic light probes $\cdot$ Monoclonal antibodies · Molecular imaging

\section{Introduction}

Gastric cancer is the fifth most common cancer and the third leading cause of cancer death worldwide, accounting for $8.8 \%$ of all cancer-related deaths every year [1]. Although overall survival of gastric cancer patients is improved with surgery including D2 lymphadenectomy and chemotherapy, including new targeted drugs, the long-term survival rates

Electronic supplementary material The online version of this article (https://doi.org/10.1007/s10120-018-0871-5) contains supplementary material, which is available to authorized users.

Hisataka Kobayashi

kobayash@mail.nih.gov

1 Molecular Imaging Program, Center for Cancer Research, National Cancer Institute, National Institutes of Health, Bethesda, MD 20892, USA are still unsatisfactory [2-4]. Peritoneal dissemination (PD) is common and occurs synchronously with detection of the primary tumor in $14-43 \%$ of patients [5]. Peritoneal recurrence is seen in $10-54 \%$ of all patients with gastric cancer after a curative surgery $[4,6]$. The prognosis for PD of gastric cancer is extremely poor and the mean survival is only 4 months after diagnosis because no effective therapies have been established [5].

Recent advances in the molecular phenotyping of gastric cancer have helped to identify predictive biomarkers that can help direct therapy. For instance, approximately $20 \%$ of gastric cancers are human epidermal growth factor receptor (EGFR) type 2 (HER2) positive $[2,7]$. Therefore, trastuzumab, an anti-HER2 monoclonal antibody (mAb), has been successfully used to treat HER2-positive gastric cancer [2]. However, its therapeutic efficacy is still limited for patients with PD. Thus, novel antitumor strategies are 
urgently required to improve the clinical outcomes for gastric cancer patients, particularly those with PD.

Near infrared photoimmunotherapy (NIR-PIT) is a newly developed cancer treatment that employs a highly targeted mAb-photo-absorber conjugate (APC) [8]. The APC binds to the appropriate cell surface antigen and the photo-activatable silica-phthalocyanine dye, IRDye700DX (IR700), is excited by NIR light leading to lethal damage to the cell membrane in a highly selective manner. NIR-PIT has been shown to be effective with a variety of different antibodies conjugated to the same IR700 dye [9-15]. A first-in-human phase 1 trial of EGFR-targeted NIR-PIT in patients with inoperable head and neck cancer was initiated in June 2015 (https://clinicaltrials.gov/ct2/show/NCT02422979) and has recently advanced to phase 2 .

The therapeutic effects of NIR-PIT depend on access of the APC to the target tissue and the ability to deliver NIR light. NIR light can be easily delivered to lesions in the mouth and skin by means of an external light source. On the other hand, an obvious limitation of NIR-PIT is the inability to deliver NIR light to tumors located deep to the surface such as intraperitoneal cancers. NIR light can penetrate several centimeters into tissue [16], but eventually skin, fat and other organs will absorb the light, making it ineffectual for therapy. One of the solutions to this problem is to introduce light fibers with diffusers, interstitially within or nearby tumors using hollow transparent catheters with needle trocars. Once placed, the trocar is removed and the optical fiber diffuser can be inserted. In the previous work, we demonstrated that the diffuser delivered sufficient NIR light dose for performing effective NIR-PIT to xenograft tumors in a mouse model [17]. Thus, NIR light administered to the intraperitoneal cavity via optical fiber diffuser could deliver sufficient light to peritoneally disseminated cancers to treat with NIR-PIT. In this study, we investigate the efficacy of NIR-PIT using a fiber optic diffuser for treating PD in a gastric cancer model.

\section{Materials and methods}

\section{Reagents}

Water soluble, silica-phthalocyanine-derivative, IRDye 700DX NHS ester was obtained from LI-COR Biosciences (Lincoln, NE, USA). Trastuzumab, 95\% humanized $\mathrm{IgG}_{1}$ $\mathrm{mAb}$ directed against HER2, was purchased from Genentech (South San Francisco, CA, USA). All other chemicals were of reagent grade.

\section{Synthesis of IR700-conjugated trastuzumab}

Conjugation of dye with $\mathrm{mAb}$ was performed according to a previous report [8]. In brief, trastuzumab $(1.0 \mathrm{mg}, 6.8 \mathrm{nmol})$ was incubated with IR700 NHS ester $(60.2 \mu \mathrm{g}, 30.8 \mathrm{nmol})$ in $0.1 \mathrm{M} \mathrm{Na}_{2} \mathrm{HPO}_{4}(\mathrm{pH} \mathrm{8.6)}$ at room temperature for $1 \mathrm{~h}$. The mixture was purified with a Sephadex G25 column (PD-10; GE Healthcare, Piscataway, NJ, USA). The protein concentration was determined with Coomassie Plus protein assay kit (Thermo Fisher Scientific Inc, Rockford, IL, USA) by measuring the absorption at $595 \mathrm{~nm}$ with UV-Vis (8453 Value System; Agilent Technologies, Santa Clara, CA, USA). The concentration of IR700 was measured by absorption at $689 \mathrm{~nm}$ with spectroscopy to confirm the number of fluorophore molecules conjugated to each $\mathrm{mAb}$. The synthesis was controlled so that an average of two IR700 molecules was bound to a single antibody. We abbreviate IR700 conjugated to trastuzumab as tra-IR700.

\section{Cell culture}

N87GFP cells stably expressing green fluorescence protein (GFP) were purchased from ANTI CANCER (San Diego, CA, USA). Luciferase-expressing N87-GFP cell was established by transducing them with RediFect Red-FLucPuromycin Lentiviral Particles (PerkinElmer, Waltham, MA, USA). Their high GFP expression and high luciferase expression was confirmed in the absence of a selection agent after 10 passages. We abbreviate this cell line as N87GFPluc. Cells were grown in RPMI 1640 (Life Technologies, Gaithersburg, MD, USA) supplemented with $10 \%$ fetal bovine serum and 1\% penicillin/streptomycin (Life Technologies) in tissue culture flasks in a humidified incubator at $37{ }^{\circ} \mathrm{C}$ in an atmosphere of $95 \%$ air and $5 \%$ carbon dioxide.

\section{Flow cytometry}

N87GFP-luc cells $\left(4 \times 10^{5}\right)$ were seeded into 12 -well plates and incubated for $24 \mathrm{~h}$. Medium was replaced with fresh culture medium containing $10 \mu \mathrm{g} / \mathrm{mL}$ of tra-IR700 and incubated for $6 \mathrm{~h}$ at $37^{\circ} \mathrm{C}$. To validate the specific binding of the conjugated antibody, excess antibody $(100 \mu \mathrm{g})$ was used to block $10 \mu \mathrm{g}$ of APCs. To verify in vitro tra-IR700 binding, fluorescence from cells after incubation with tra-IR700 was measured using a flow cytometer (FACS Calibur, BD BioSciences, San Jose, CA, USA) and CellQuest software (BD BioSciences). A 635-nm red diode laser was used for excitation. Signals from cells were collected with a 653-669-nm filter. 


\section{Fluorescence microscopy}

To detect the antigen-specific localization and effect of NIR-PIT, fluorescence microscopy was performed (BX61; Olympus America, Inc., Melville, NY, USA). Ten thousand N87GFP-luc cells were seeded on cover-glass-bottomed 6-well dishes and incubated for $24 \mathrm{~h}$. Tra-IR700 was then added to the culture medium at $10 \mu \mathrm{g} / \mathrm{mL}$ and incubated for $6 \mathrm{~h}$ at $37{ }^{\circ} \mathrm{C}$. After incubation, the cells were washed with phosphate buffered saline (PBS). The filter set to detect IR700 consisted of a 590-650-nm excitation filter, a 665-740-nm band pass emission filter. Transmitted light differential interference contrast (DIC) images were also acquired.

\section{In vitro NIR-PIT}

The cytotoxic effects of NIR-PIT for N87GFP-luc cell with tra-IR700 were determined by flow cytometric propidium iodide (PI) (Life Technologies, Gaithersburg, MD, USA) staining, which can detect compromised cell membranes. Four hundred thousand N87GFP-luc cells were seeded into 12-well plates and incubated for $24 \mathrm{~h}$. Medium was replaced with fresh culture medium containing $10 \mu \mathrm{g} / \mathrm{mL}$ of tra-IR700 and incubated for $6 \mathrm{~h}$ at $37{ }^{\circ} \mathrm{C}$. After washing with PBS, PBS was added, and cells were irradiated with a red light-emitting diode (LED), which emits light at $690 \pm 20 \mathrm{~nm}$ wavelength (L690-66-60; Marubeni America Co., Santa Clara, CA, USA) at a power density of $50 \mathrm{~mW} /$ $\mathrm{cm}^{2}$ as measured with an optical power meter (PM 100, Thorlabs, Newton, NJ, USA). Cells were scratched $1 \mathrm{~h}$ after treatment. PI was then added to the cell suspension (final $2 \mu \mathrm{g} / \mathrm{mL}$ ) and incubated at room temperature for $30 \mathrm{~min}$, followed by flow cytometry. A 488-nm argon ion laser was used for excitation. Signals from cells were collected with a 515-545-nm band-pass filter and a 650-nm long-pass filter for GFP and PI, respectively.

For bioluminescence imaging (BLI), either two hundred thousand N87GFP-luc cells were seeded into 12-well plates or twenty million N87GFP-luc cells were seeded onto a 10 -cm dish; both were pre-incubated for $24 \mathrm{~h}$. After replacing the medium with fresh culture medium containing $10 \mu \mathrm{g} /$ $\mathrm{mL}$ of tra-IR700, the cells were incubated for $6 \mathrm{~h}$ at $37^{\circ} \mathrm{C}$ in a humidified incubator. After washing with PBS, phenol-redfree culture medium was added. Then, cells were exposed with a LED or a NIR laser which emits light at $690 \pm 5-\mathrm{nm}$ wavelength (BWF5-690-8-600-0.37; B\&W TEK INC., Newark, DE, USA). The output power density in $\mathrm{mW} / \mathrm{cm}^{2}$ was also measured with an optical power meter (PM 100, Thorlabs, Newton, NJ, USA). For luciferase activity, $150 \mu \mathrm{g} /$ mL D-luciferin (Gold Biotechnology, St. Louis, MO, USA)containing media was administered to PBS-washed cells $1 \mathrm{~h}$ after NIR-PIT which were analyzed on a BLI system (Photon
Imager; Biospace Lab, Paris, France), with luciferase activity (photons/min). Regions of interest (ROIs) were placed over each well, and the sum of luciferase activity was then calculated using M3 Vision Software (Biospace Lab).

\section{Animal and tumor models}

All in vivo procedures were conducted in compliance with the Guide for the Care and Use of Laboratory Animal Resources (1996), US National Research Council, and approved by the local Animal Care and Use Committee. 6-8-week-old female homozygote athymic nude mice were purchased from Charles River (NCI-Frederick, Frederick, MD, USA). During the procedure, mice were anesthetized with inhaled $3-5 \%$ isoflurane and/or via intraperitoneal injection of $1 \mathrm{mg}$ of sodium pentobarbital (Nembutal Sodium Solution, Ovation Pharmaceuticals Inc., Deerfield, IL, USA). Body weight was measured twice a week. Mice were monitored daily for their general health. The presence of skin necrosis or toxicity attributable to the APC was evaluated by carefully noting skin color, general health, weight loss and loss of appetite.

\section{In vivo fluorescence endoscopy}

Fluorescence endoscopic devices used in this study are shown in Fig. 1a. A model BF TYPE P60 bronchoscope (Olympus Medical Systems, Tokyo, Japan), which has a $4.9 \mathrm{~mm}$ distal end diameter, $5.0 \mathrm{~mm}$ insertion tube diameter and $2.2 \mathrm{~mm}$ channel diameter with a single biopsy channel, was inserted into the abdominal cavity through a small abdominal incision, and the abdominal cavity was inflated with air (Fig. 1b). The surface of the peritoneum was observed with white light and fluorescence imaging using a clinical endoscopic light source (CLV-S190, Olympus Medical Systems, Tokyo, Japan) equipped with multiband excitation filter. Endoscopic images were obtained via a beam splitter, where the white light images were detected using the color-charge-coupled device (CCD) camera (FLIR Integrated Imaging Solutions, BC, Canada) and the fluorescence images were filtered by emission filters (507-527-nm band pass for GFP and 696-736-nm band pass for IR700) and detected with an (EM)-CCD camera (HAMAMATSU PHOTONICS, Shizuoka, Japan). Both images were displayed side by side on the PC monitor. Real-time images of both white light and fluorescence images were recorded. To compare fluorescence intensities during NIR-PIT, the distance between the tumor and the endoscope head was maintained using the biopsy forceps (FB-56D-1, Olympus Medical Systems, Tokyo, Japan) as a guide. Camera gain, exposure time, and binning for the fluorescence images were held constant throughout the study. 
a

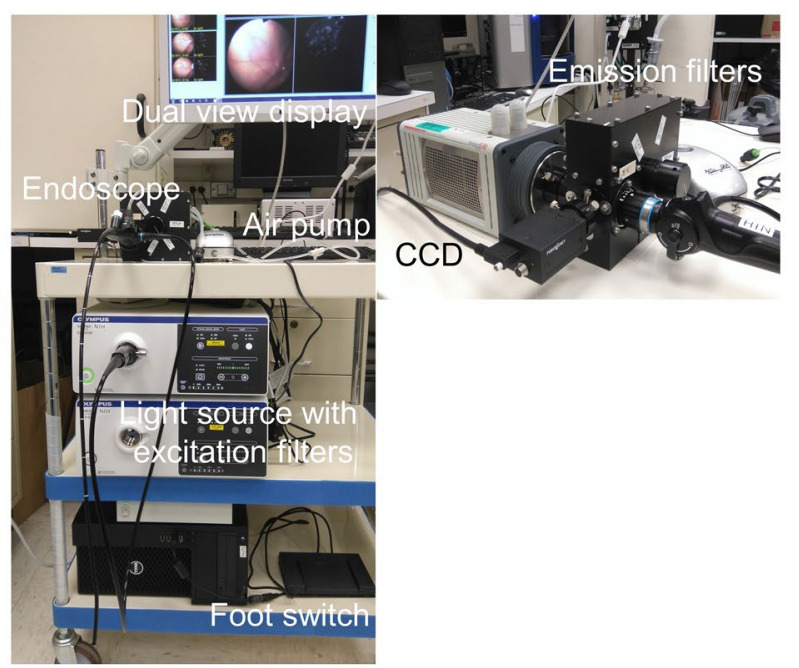

b

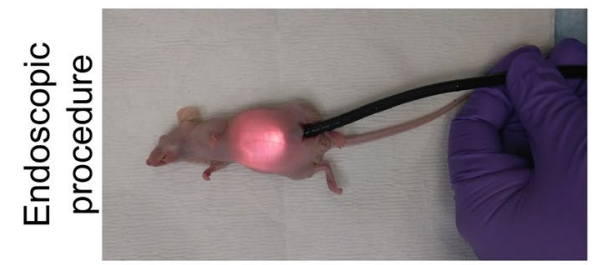

C

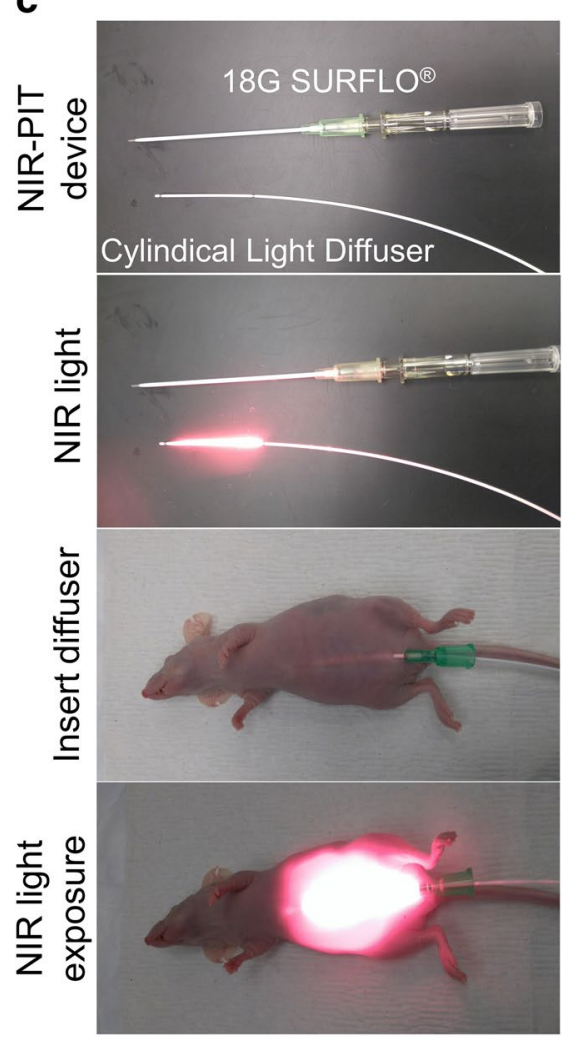

Fig. 1 Multi-color fluorescence mini-endoscopic system and NIR-PIT procedure. a Multi-color fluorescence imaging system is based on a clinically available fiber optic endoscope and light source. Excitation light is provided by multi-band excitation filters. Endoscopic images were obtained via a beam splitter, where the white light images were detected using the color-CCD camera and the fluorescence images were filtered by multi-color emission filters and detected with an

\section{Histological analysis}

Real-time tumor biopsies were performed through the fluorescence endoscope using biopsy forceps (FB-56D-1, Olympus Co., Tokyo, Japan) beginning $6 \mathrm{~h}$ after NIR-PIT (Supplementary Video 1). We could not observe the longterm course (days) in the mice because the endoscopic system used proved too invasive for mice. From this point, we selected this time course. Mice were euthanized with an overdose of carbon dioxide after the biopsies. Biopsy specimens were fixed for at least $24 \mathrm{~h}$ with $10 \%$ formalin. Paraffin-embedded sections were stained with hematoxylin and eosin (H\&E). Post NIR-PIT tissue analysis was performed using a fluorescence microscope (BX61; Olympus America, Inc., Melville, NY, USA).

\section{In vivo NIR-PIT}

To generate the disseminated peritoneal cancer mouse model, 10 million N87GFP-luc cells with PBS (total $400 \mu \mathrm{L}$ )
(EM)-CCD camera. Both images are displayed side by side on the monitor. b The endoscope was inserted into abdominal cavity through a small lower abdominal incision, and the abdominal cavity was inflated with air. c Peritoneal cavity was exposed to NIR light using a Cylindrical Light Diffuser with a NIR laser through the $18 \mathrm{G} \times 21 / 2^{\prime \prime}$ SURFLO®

were injected into the peritoneal cavity. Mice developed disseminated peritoneal cancer at 8 days after cell implantation and were randomized into 5 groups of at least 10 animals per group for the following treatments: (1) no treatment (control); (2) $100 \mu \mathrm{g}$ of tra-IR700 i.v., no NIR light exposure, this therapy was performed every week for up to 3 weeks (APC i.v. only); (3) NIR light exposure only, NIR light was administered at $50 \mathrm{~J} / \mathrm{cm}$ on day 1 and $100 \mathrm{~J} / \mathrm{cm}$ on day 2 , this therapy was performed every week for up to 3 weeks (NIR light only); (4) $100 \mu \mathrm{g}$ of tra-IR700 i.v., NIR light was administered at $50 \mathrm{~J} / \mathrm{cm}$ on day 1 and $100 \mathrm{~J} / \mathrm{cm}$ on day 2 after tra-IR700 injection. This therapy was performed once (NIR-PIT); (5) $100 \mu \mathrm{g}$ of tra-IR700 i.v., NIR light was administered at $50 \mathrm{~J} / \mathrm{cm}$ on day 1 and $100 \mathrm{~J} / \mathrm{cm}$ on day 2 after tra-IR700 injection, this therapy was performed every week for up to 3 weeks (repeated NIR-PIT). Peritoneal cavity was irradiated using a fiber optic diffuser (Cylindrical Light Diffuser model RD30; Medlight, Ecublens, Switzerland) inserted into a catheter $\left(18 \mathrm{G} \times 21 / 2^{\prime \prime}\right.$ SURFLO®; TERUMO, Somerset, NJ, USA) and coupled to a NIR laser 
which emits light at 685-695 nm (BWF5-690-8-600-0.37; B\&W TEK INC., Newark, DE, USA) (Fig. 1c). The output power density in $\mathrm{mW} / \mathrm{cm}$ was measured with an optical power meter (PM 100, Thorlabs, Newton, NJ, USA).

For in vivo BLI, D-luciferin $(15 \mathrm{mg} / \mathrm{mL}, 200 \mu \mathrm{L})$ was injected intraperitoneally and the mice were imaged (Photon Imager) for luciferase activity (photons/min). ROIs were set on the entire tumor to quantify the light flux. ROIs were also placed on adjacent non-tumor regions as background. Average light flux of each ROI was calculated.

\section{Statistical analysis}

Data are expressed as means \pm standard error of mean (SEM) from a minimum of five experiments, unless otherwise indicated. Statistical analyses were carried out using GraphPad Prism version 7 (GraphPad Software, La Jolla, CA, USA). For multiple comparisons, a one-way analysis of variance (ANOVA) followed by the Tukey's correction for multiple comparisons was used. For survival, Kaplan-Meier survival curve analysis, and the results were compared with use of the log-rank test. Student's $t$ test was used to compare the in vitro treatment effects with that of control. A $p$ value of $<0.05$ was considered statistically significant.

\section{Results}

\section{In vitro characterization of N87GFP-luc cell}

After a $6 \mathrm{~h}$ incubation with tra-IR700, N87GFP-luc cells demonstrated high fluorescence signal, which was confirmed with flow cytometry (Fig. 2a) and fluorescence microscopy (Fig. 2b). On the other hand, N87GFP-luc cells without incubation with tra-IR700 showed no fluorescence signal (Fig. 2b). Fluorescence signal in N87GFP-luc cells was completely blocked by adding excess trastuzumab (Fig. 2a), indicating that the APC specifically binds to EGFR on the N87GFP-luc cells.

\section{In vitro NIR-PIT}

N87GFP-luc cells not incubated with tra-IR700 showed no obvious change after NIR light exposure (Fig. 2b). N87GFPluc cells incubated with tra-IR700 showed immediate swelling, bleb formation, and rupture of vesicles after the exposure to NIR light (Supplementary Video 2). Most of these morphologic changes were observed within $15 \mathrm{~min}$ of light exposure (Fig. 2b). BLI showed significant decreases of luciferase activity in NIR-PIT-treated cells (Fig. 2c, d). Based on incorporation of PI, the percentage of cell death increased in a light dose-dependent manner in N87GFP-luc cells (Fig. 2e). Over $80 \%$ of N87GFP-luc cells died when exposed to $8 \mathrm{~J} / \mathrm{cm}^{2}$ of NIR light. Furthermore, GFP fluorescence was reduced after NIR-PIT because the GFP escaped from the cytoplasm after cell membrane rupture and was, therefore, markedly diluted and/or denatured. As a result, GFP diminished within cells in a light dose-dependent manner (Fig. 2f). There was no significant cytotoxicity associated with NIR light alone in the absence of APC and with APC alone without NIR light.

\section{In vivo fluorescence endoscopy and histological analysis}

Prior to in vivo NIR-PIT, implanted intraperitoneal disseminated tumors were evaluated with fluorescence endoscopy. The treatment and imaging regimen are shown in Fig. 3a. Fluorescence endoscopy demonstrated multiple 1-3 mm intraperitoneal disseminated small nodule establishment based on GFP fluorescence. After tra-IR700 injection, the implanted intraperitoneal disseminated tumors demonstrated fluorescence commensurate with IR700 which co-localized with GFP (Fig. 3b; APC i.v. only and NIR-PIT group). On the other hand, no IR700 fluorescence signal was observed in tumors without tra-IR700 injection (Fig. 3b; control and NIR light only group). These results demonstrate that intravenous injection of tra-IR700 was able to bind to intraperitoneal implants. In animals undergoing NIR-PIT, IR700 fluorescence signal in tumors diminished due to a combination of dead cells no longer binding tra-IR700 and partial photo-bleaching of IR700 (Fig. 3b).

H\&E staining of NIR-PIT-treated N87GFP-luc tumors revealed diffuse necrosis and micro-hemorrhage, with scattered clusters of live but damaged tumor cells, while no obvious damage was observed in any of the control groups (Fig. 3c).

\section{In vivo NIR-PIT}

The treatment and imaging regimen of NIR-PIT are shown in Fig. 4a. After treatment with NIR-PIT intraperitoneal disseminated N87GFP-luc tumors decreased in bioluminescence (Fig. 4b). Bioluminescence significantly decreased in all three treatment groups (tra-IR700 i.v. only, NIR-PIT, and repeated NIR-PIT group) compared with control and NIR light only group $(p<0.001$ for tra-IR700 i.v. only group, $p<0.0001$ for NIR-PIT group and repeated NIR-PIT group). Both NIR-PIT and repeated NIR-PIT groups showed significantly lower bioluminescence than the tra-IR700 i.v. only group ( $p<0.001$ for NIR-PIT group, $p<0.0001$ for repeated NIR-PIT group). The group demonstrating the best therapeutic effect was the repeated NIR-PIT group ( $p<0.01$ vs NIR-PIT group) (Fig. 4c). After NIR-PIT, significant loss of body weight was observed in NIR-PIT and repeated NIRPIT groups; however, the mice recovered weight over the 
a

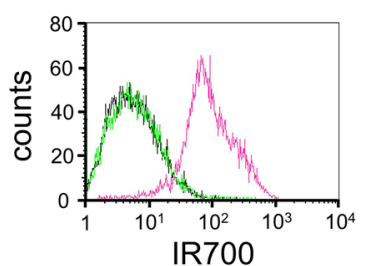

-N87GFP-luc control

-N87GFP-luc + tra-IR700

-N87GFP-luc + tra-IR700

+ blocking b

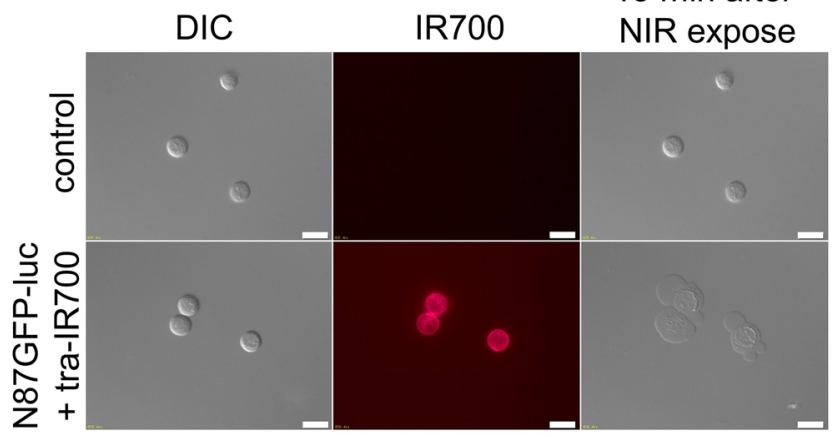

C

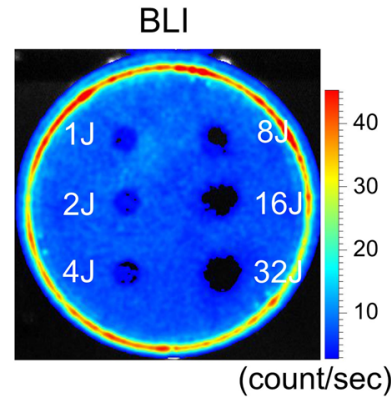

d

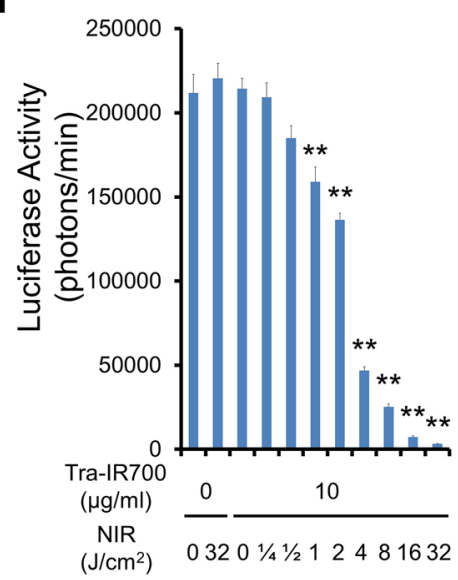

e

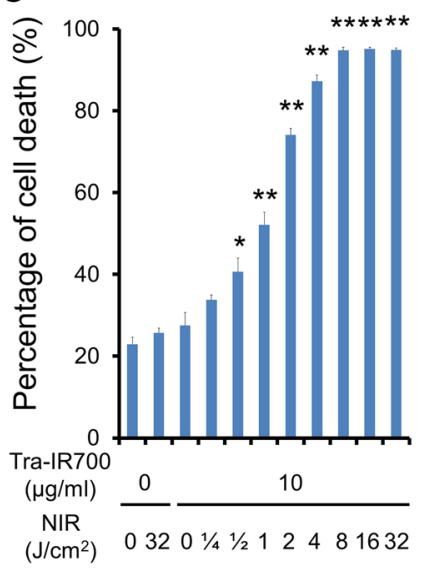

f

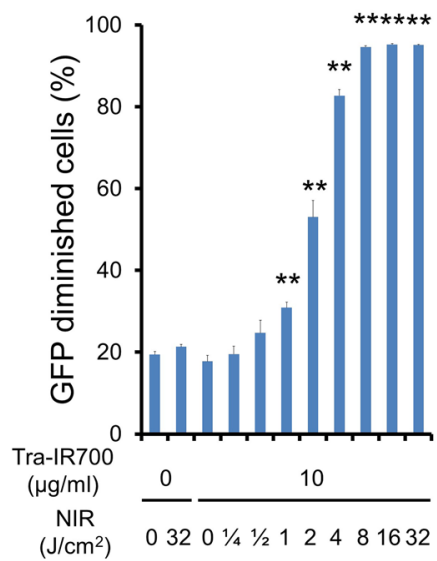

Fig. 2 Confirmation of HER2 expression as a target for NIR-PIT in N87GFP-luc cells, and evaluation of in vitro NIR-PIT. a Expression of HER2 in N87GFP-luc cells was examined with FACS. After $6 \mathrm{~h}$ of tra-IR700 incubation, N87GFP-luc cells showed high fluorescence signal. b Differential interference contrast (DIC) and fluorescence microscopy images of N87GFP-luc cells. High IR700 fluorescence signal was shown in N87GFP-luc cells after incubation with traIR700 for $6 \mathrm{~h}$. On the other hand, there was no fluorescence signal in N87GFP-luc cells without incubation with tra-IR700. Immunogenic/ necrotic cell death was observed upon excitation with NIR light (after $15 \mathrm{~min}$ ) only in N87GFP-luc cells incubated with tra-IR700. Scale bars $=20 \mu \mathrm{m}$. c Bioluminescence imaging (BLI) of a $10-\mathrm{cm}$ dish demonstrated that luciferase activity in N87GFP-luc cells decreased in a NIR-light dose-dependent manner. d Luciferase activity in N87GFP-luc cells was measured, which also decreased in a NIR-light dose-dependent manner $(n=5, * * p<0.01$ vs untreated control, by Student's $t$ test). e Membrane damage of N87GFP-luc cells induced by NIR-PIT was measured with propidium iodide (PI) staining, which increased in a light dose-dependent manner $(n=5, * p<0.05$, $* * p<0.01$, vs. untreated control, by Student's $t$ test). f Diminishing GFP fluorescence intensity in N87GFP-luc cells after NIR-PIT was measured by FACS. The GFP diminished within cells in a light dosedependent manner $(n=5, * * p<0.01$, vs. untreated control, by Student's $t$ test) following week (Fig. 4d). Significantly prolonged survival was also achieved in the three treatment groups (tra-IR700 i.v. only, NIR-PIT, and repeated NIR-PIT group) compared with the control and NIR light only group $(p<0.05$ for tra-IR700 i.v. only group, $p<0.01$ for NIR-PIT group, and $p<0.0001$ for repeated NIR-PIT group). Repeated NIRPIT group showed significantly longer survival than the traIR700 i.v. only group and the NIR-PIT group $(p<0.0001$ vs tra-IR700 i.v. only group, $p<0.01$ vs NIR-PIT group) (Fig. 4e). No significant therapeutic effect was observed in the mice receiving NIR light only. No skin damage was observed attributable to NIR-PIT in any group.

\section{Discussion}

Despite recent advances in chemotherapy and molecularly targeted therapies directed against advanced gastric cancer, the prognosis of patients with disseminated peritoneal gastric cancer is poor [5]. In this study, we showed that NIR-PIT administered via a fiber optic diffuser successfully reduces tumor burden and improves survival in a mouse model of HER2-positive disseminated peritoneal gastric cancers. This application is readily translated into clinical use.

The APC used in this study, tra-IR700, demonstrated excellent binding and microdistribution within the 
a

\begin{tabular}{|c|c|c|c|c|}
\hline & -24 & 0 & 6 & hrs \\
\hline 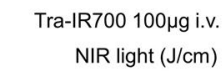 & $\uparrow$ & 50 & & \\
\hline orescence endoscope & & & $\begin{array}{c}\uparrow \\
\text { Biopsy }\end{array}$ & \\
\hline
\end{tabular}

b

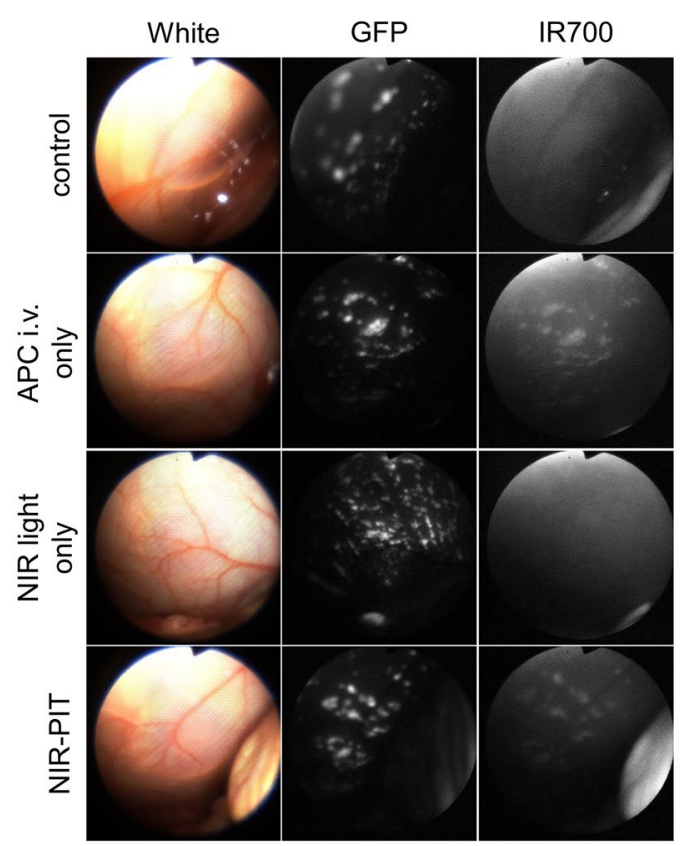

C

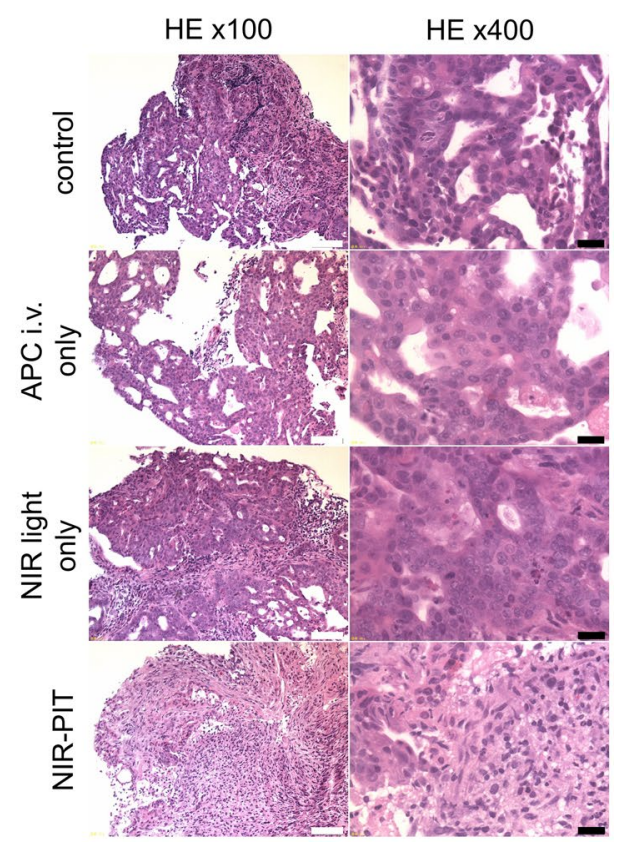

Fig. 3 In vivo fluorescence real-time endoscopic imaging and histological NIR-PIT effect. a Treatment regimen is shown. Biopsy specimens were obtained $6 \mathrm{~h}$ after NIR-PIT. b In vivo fluorescence real-time endoscopic intraperitoneal imaging of N87GFP-luc tumorbearing mice. Tumors demonstrate GFP and IR700 fluorescence in tra-IR700 injected tumors. In the absence of tra-IR700 injection no IR700 fluorescence was seen. After NIR-PIT IR700 fluorescence

HER2-expressing peritoneal gastric cancer implants after intravenous injection. The conjugation of IR700 to trastuzumab did not influence the binding affinity of the antibody or limit its distribution. As a result, tra-IR700 demonstrated high target accumulation and specific killing of HER2-positive gastric cancer cells after NIR light exposure (Fig. 2 and Supplementary Video 2).

There are several preconditions for successful NIR-PIT in vivo. First, there must be adequate uptake and retention of the APC in the target tumor. In vivo fluorescence endoscopy clearly showed IR700 fluorescence within peritoneal implants $24 \mathrm{~h}$ after intravenous injection of tra-IR700 (Fig. 3). Another pre-requisite for successful NIR-PIT is the ability to deliver NIR light. In the clinical situation, peritoneal implants would have to be exposed to NIR light either during an open surgery or via fiber optic laparoscopy. The latter is less invasive and likely to lead to a faster patient recovery. As shown in Fig. 1, a fiber optic diffuser could be transcutaneously introduced decreased. c Biopsy specimens stained with hematoxylin and eosin (H\&E). A few scattered clusters of damaged tumor cells are seen within a background of diffuse cellular necrosis and micro-hemorrhage, while no obvious damage was observed in control groups, including tra-IR700 alone without NIR light and NIR light alone without tra-IR700 tumors. White scale bars $=100 \mu \mathrm{m}$. Black scale bars $=20 \mu \mathrm{m}$

into the peritoneal cavity via a catheter or laparoscope. Similarly, the fiber optic light diffuser could be placed within or near tumor using endoscope, laparoscope or image-guided percutaneous needles. Since most intraperitoneal implants do not exceed $2 \mathrm{~cm}$ in thickness, this light exposure could effectively treat peritoneal implants. Additionally, NIR-PIT will effectively treat floating cancer cells in ascites that promote intraperitoneal implants because APC could distribute in ascites and well bind to such cells.

NIR-PIT results in acute cellular necrosis and microhemorrhage (Fig. 3). Cell death is confirmed by loss of BLI signal (Fig. 4). Loss of IR700 fluorescence implies both cell death and photo-bleaching, a sign that NIR light has reached its target. Taken together, NIR-PIT using a fiber optic diffuser was highly effective in killing tumor implants. Even better tumor control was seen with repeated NIR-PIT treatments. The effect of tra-IR700 alone without NIR light was modest. It has been previously reported that additional 
a

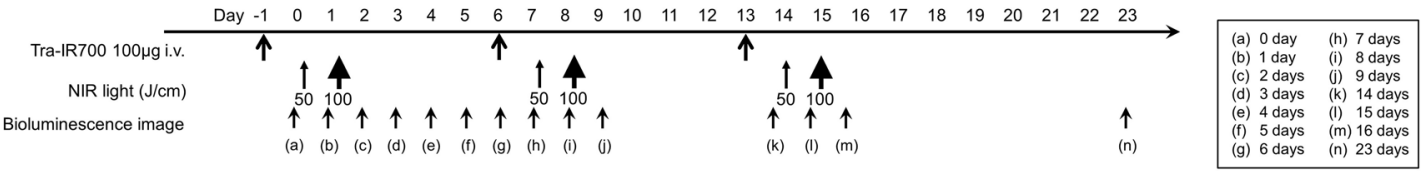

b
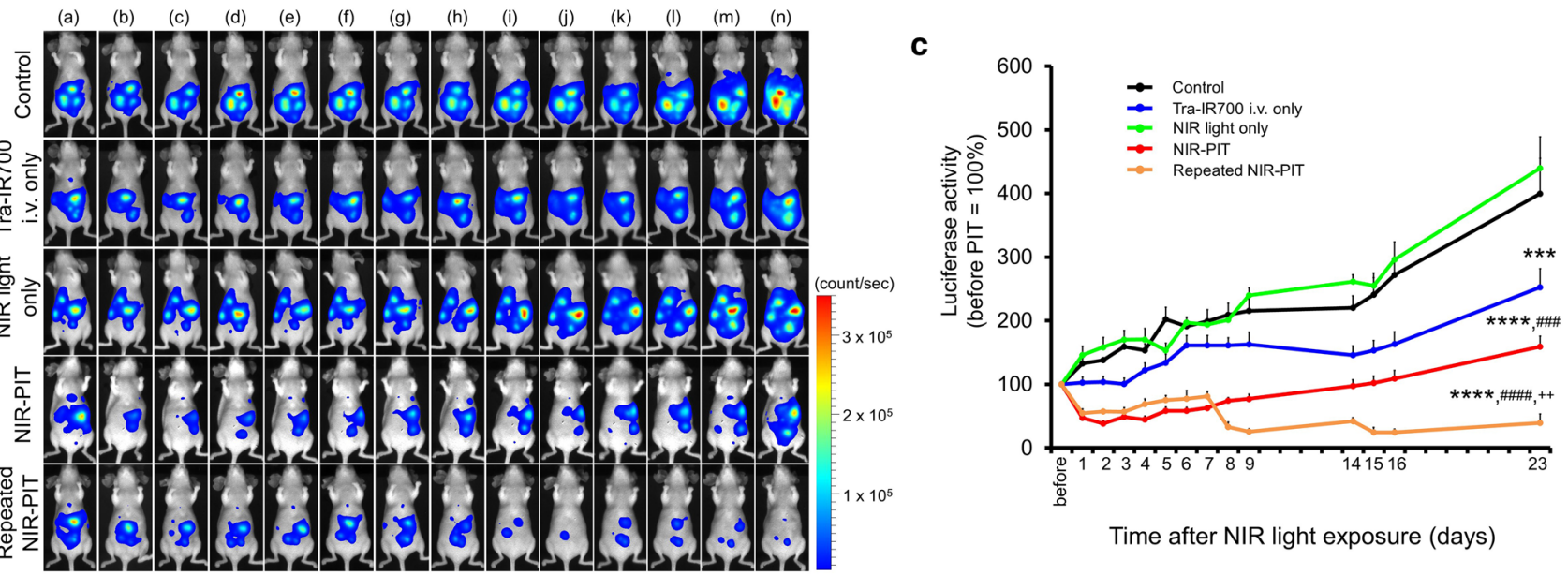

d

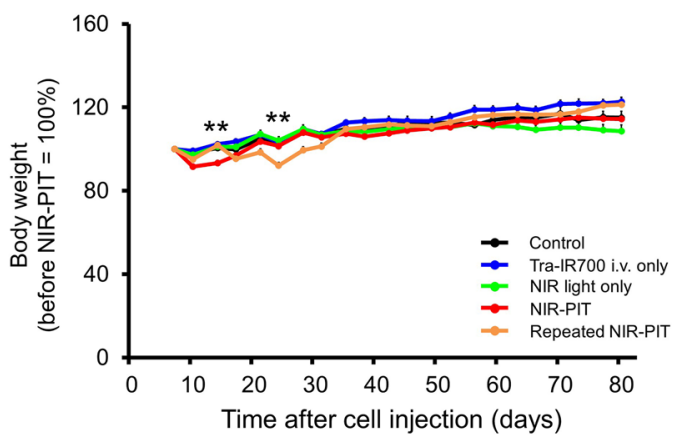

Fig. 4 In vivo effect of NIR-PIT in peritoneal N87GFP-luc tumors. a NIR-PIT regimen. Bioluminescence images were obtained at each time point as indicated. b In vivo BLI of tumor bearing mice in response to NIR-PIT. Before NIR-PIT, tumors exhibited high signal on BLI. The tumors treated by NIR-PIT showed a marked decrease in BLI. c BLI showed a significant decrease in three treatment groups (tra-IR700 i.v. only, NIR-PIT, and repeated NIR-PIT group) compared with control and NIR light only group $(n \geqq 10, * * * p<0.001$ for tra-IR700 i.v. only group, $* * * * p<0.0001$ for NIR-PIT and repeated NIR-PIT group, by Tukey's $t$ test with ANOVA). Both NIR-PIT and repeated NIR-PIT groups showed significantly decreased BLI activity than the tra-IR700 i.v. only group $\left(n \geqq 10,{ }^{\# \#} p<0.001\right.$ for NIR-

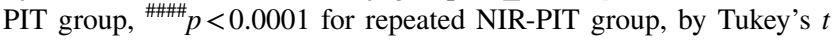

trastuzumab in standard chemotherapy has significantly increased the overall survival in the treatment of metastatic gastric cancer [2]. Furthermore, treatment of peritoneal tumors with intraperitoneal trastuzumab has also been reported [18]. From these results, it is not surprising that small therapeutic effects were seen with tra-IR700 alone.

Repeated dosing of APC and NIR light has been previously reported to improve efficacy of NIR-PIT [11, 19, 20]. We confirmed this effect in this mouse model. Interestingly, this strategy did not appear to result in treatment resistance.

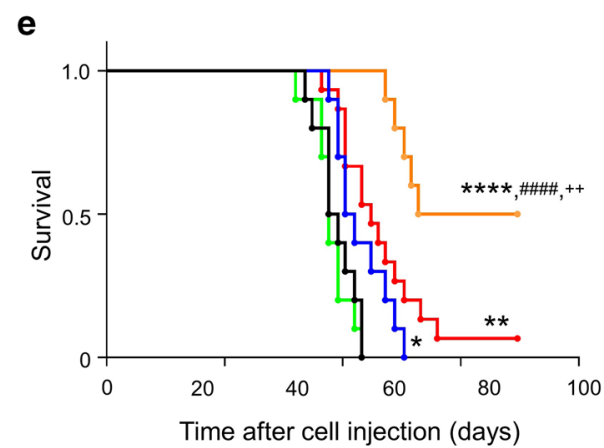

test with ANOVA). Most therapeutic effect was shown in repeated NIR-PIT group $\left(n \geqq 10,{ }^{++} p<0.01\right.$ vs NIR-PIT group, by Tukey's $t$ test with ANOVA). d After NIR-PIT, significant loss of body weight was observed in the NIR-PIT and repeated NIR-PIT groups $(n \geqq 10$, ${ }^{* *} p<0.01$ vs other groups, by Tukey's $t$ test with ANOVA); however, the body weight recovered gradually. e Significantly prolonged survival was observed in the three treatment groups (tra-IR700 i.v. only, NIR-PIT, and repeated NIR-PIT group) compared with control and NIR light only groups $(n \geqq 10, * p<0.05$ for tra-IR700 i.v. only group, $* * p<0.01$ for NIR-PIT, and $* * * * p<0.0001$ for repeated NIR-PIT group). Repeated NIR-PIT group showed significantly prolonged survival compared to the tra-IR700 i.v. only group ( $n \geqq 10$, $\#$ \#\#\# $p<0001)$ and NIR-PIT group $\left(n \geqq 10,{ }^{++} p<0.01\right)$

NIR-PIT should not be confused with intraperitoneal photodynamic therapy (PDT) which has been previously tested in patients after surgical debulking of disseminated intraperitoneal tumors [21]. However, unlike NIR-PIT, PDT with a dihematoporphyrin ether derivative produces substantially more toxicity due to non-specificity of the photosensitizer. Recently, PDT using various types of photosensitizing agents thought to target PD of gastric cancer have been reported [22, 23]. Unfortunately, due to the hydrophobic nature of the porphyrin photosensitizers, they are difficult 
to target and this diminishes the potential selectivity of the agent. On the other hand, the hydrophilic phthalocyaninebased photosensitizer, IR700DX, which is used in NIR-PIT is readily directed to the target via the antibody. PDT is activated by visible range light and thus has limited penetrance in tissue while NIR-PIT is activated by NIR light with deeper tissue penetration.

This study has several limitations. First, not all PD of gastric cancers overexpress HER2 [2, 7] and, therefore, this particular target may not be ideal for all disseminated peritoneal gastric cancer implants. Furthermore, tumors growing in patients are generally heterogeneous [24, 25]. However, NIR-PIT has proven effective with a variety of antibodies with which it has been attempted and, therefore, it is likely that the proper APC or combination of APCs could be found to treat a specific phenotype of gastric cancer [10, 12, 26]. Repeated NIR-PIT with combinations of different APCs would improve therapeutic effects to a heterogeneous cancer because NIR-PIT is minimally invasive and can be used repeatedly without dose limitation that could overcome treatment resistance [27]. Moreover, it is possible that HER2 extracellular domain could be transfected into disseminated cancers using and adenoviral HER2-extracellular domain gene transfer as has been demonstrated in mice [28]. This method has the potential to add back membrane antigens that are lacking in a particular cancer. Another caveat of this study is that we used BLI to monitor tumor growth of PD. Since NIR-PIT induces necrotic/immunogenic cell death which releases ATP [29]. BLI is an appropriate and sensitive biomarker for monitoring the therapeutic effect of NIR-PIT [30, 31]. BLI while a well-established method in mouse models, it cannot be used in humans. For this purpose, we have previously shown that ${ }^{18} \mathrm{~F}-\mathrm{FDG}$ positron emission tomography (PET) can depict the acute cytotoxic effects of NIR-PIT due to the shutting down of glucose metabolism [32]. New methods are needed to monitor the realtime effects of NIR-PIT. Finally, this study was also done in an immune-incompetent mouse model. As the immune effects of NIR-PIT are currently unclear it is difficult to know whether the effects will be amplified in immune-intact patients. Preliminary data suggest that the release of signaling molecules such as calreticulin, ATP, and high mobility group box 1 (HMGB1) strongly suggest the ability of NIRPIT to effectively promote maturation of immature dendritic cells and, therefore, activate a strong immune response that will augment the direct cytotoxicity of NIR-PIT [33].

\section{Conclusion}

To perform effective NIR-PIT for peritoneal disseminated gastric cancer, the APC should be delivered intravenously and NIR light should be delivered with sufficient energy into the peritoneal cavity. If these conditions are met, NIR-PIT using tra-IR700 induced remarkable therapeutic responses especially after repeated NIR-PIT. Thus, NIR-PIT could be a promising adjuvant for treating PD of gastric cancer using fiber optic diffusers to deliver the activating light.

Funding All authors were supported by the Intramural Research Program of the NIH, NCI, Center for Cancer Research.

\section{Compliance with ethical standards}

Conflict of interest No potential conflicts of interest were disclosed.

Ethical standards All institutional and national guidelines for the care and use of laboratory animals were followed.

\section{References}

1. Torre LA, Bray F, Siegel RL, Ferlay J, Lortet-Tieulent J, Jemal A. Global cancer statistics, 2012. CA Cancer J Clin. 2015;65(2):87-108.

2. Bang YJ, Van Cutsem E, Feyereislova A, Chung HC, Shen L, Sawaki A, et al. Trastuzumab in combination with chemotherapy versus chemotherapy alone for treatment of HER2-positive advanced gastric or gastro-oesophageal junction cancer (ToGA): a phase 3, open-label, randomised controlled trial. Lancet. 2010;376(9742):687-97.

3. Noh SH, Park SR, Yang HK, Chung HC, Chung IJ, Kim SW, et al. Adjuvant capecitabine plus oxaliplatin for gastric cancer after D2 gastrectomy (CLASSIC): 5-year follow-up of an open-label, randomised phase 3 trial. Lancet Oncol. 2014;15(12):1389-96.

4. Sasako M, Sakuramoto S, Katai H, Kinoshita T, Furukawa $\mathrm{H}$, Yamaguchi $\mathrm{T}$, et al. Five-year outcomes of a randomized phase III trial comparing adjuvant chemotherapy with S-1 versus surgery alone in stage II or III gastric cancer. J Clin Oncol. 2011;29(33):4387-93.

5. Thomassen I, van Gestel YR, van Ramshorst B, Luyer MD, Bosscha K, Nienhuijs SW, et al. Peritoneal carcinomatosis of gastric origin: a population-based study on incidence, survival and risk factors. Int J Cancer. 2014;134(3):622-8.

6. Wu CW, Lo SS, Shen KH, Hsieh MC, Chen JH, Chiang JH, et al. Incidence and factors associated with recurrence patterns after intended curative surgery for gastric cancer. World J Surg. 2003;27(2):153-8.

7. Park DI, Yun JW, Park JH, Oh SJ, Kim HJ, Cho YK, et al. HER-2/ neu amplification is an independent prognostic factor in gastric cancer. Dig Dis Sci. 2006;51(8):1371-9.

8. Mitsunaga M, Ogawa M, Kosaka N, Rosenblum LT, Choyke PL, Kobayashi H. Cancer cell-selective in vivo near infrared photoimmunotherapy targeting specific membrane molecules. Nat Med. 2011;17(12):1685-91.

9. Hanaoka H, Nagaya T, Sato K, Nakamura Y, Watanabe R, Harada $\mathrm{T}$, et al. Glypican-3 targeted human heavy chain antibody as a drug carrier for hepatocellular carcinoma therapy. Mol Pharm. 2015;12(6):2151-7.

10. Nagaya T, Nakamura Y, Okuyama S, Ogata F, Maruoka Y, Choyke PL, et al. Syngeneic mouse models of oral cancer are effectively targeted by anti-CD44-Based NIR-PIT. Mol Cancer Res. 2017;15(12):1667-77.

11. Nagaya T, Nakamura Y, Okuyama S, Ogata F, Maruoka Y, Choyke PL, et al. Near-infrared photoimmunotherapy targeting prostate 
cancer with prostate-specific membrane antigen (PSMA) Antibody. Mol Cancer Res. 2017;15(9):1153-62.

12. Nagaya T, Nakamura Y, Sato K, Harada T, Choyke PL, Hodge JW, et al. Near infrared photoimmunotherapy with avelumab, an anti-programmed death-ligand 1 (PD-L1) antibody. Oncotarget. 2017;8(5):8807-17.

13. Nagaya T, Nakamura Y, Sato K, Harada T, Choyke PL, Kobayashi H. Near infrared photoimmunotherapy of B-cell lymphoma. Mol Oncol. 2016;10(9):1404-14.

14. Nagaya T, Nakamura Y, Sato K, Zhang YF, Ni M, Choyke PL, et al. Near infrared photoimmunotherapy with an anti-mesothelin antibody. Oncotarget. 2016;7(17):23361-9.

15. Sato K, Choyke PL, Kobayashi H. Photoimmunotherapy of gastric cancer peritoneal carcinomatosis in a mouse model. PLoS One. 2014;9(11):e113276.

16. Henderson TA, Morries LD. Near-infrared photonic energy penetration: can infrared phototherapy effectively reach the human brain? Neuropsychiatr Dis Treat. 2015;11:2191-208.

17. Shuhei O, Tadanob N, Kazuhide S, Fusa O, Yasuhiro M, Choyke PL, et al. Interstitial near-infrared photoimmunotherapy: effective treatment areas and light doses needed for use with fiber optic diffusers. Oncotarget. 2018;9(13):11159-69.

18. Berretta M, Fisichella R, Borsatti E, Lleshi A, Ioffredo S, Meneguzzo N, et al. Feasibility of intraperitoneal Trastuzumab treatment in a patient with peritoneal carcinomatosis from gastric cancer. Eur Rev Med Pharmacol Sci. 2014;18(5):689-92.

19. Mitsunaga M, Nakajima T, Sano K, Choyke PL, Kobayashi H. Near-infrared theranostic photoimmunotherapy (PIT): repeated exposure of light enhances the effect of immunoconjugate. Bioconjug Chem. 2012;23(3):604-9.

20. Nagaya T, Sato K, Harada T, Nakamura Y, Choyke PL, Kobayashi $\mathrm{H}$. Near infrared photoimmunotherapy targeting EGFR positive triple negative breast cancer: optimizing the conjugate-light regimen. PLoS One. 2015;10(8):e0136829.

21. DeLaney TF, Sindelar WF, Tochner Z, Smith PD, Friauf WS, Thomas G, et al. Phase I study of debulking surgery and photodynamic therapy for disseminated intraperitoneal tumors. Int J Radiat Oncol Biol Phys. 1993;25(3):445-57.

22. Hino H, Murayama $Y$, Nakanishi M, Inoue K, Nakajima M, Otsuji E. 5-Aminolevulinic acid-mediated photodynamic therapy using light-emitting diodes of different wavelengths in a mouse model of peritoneally disseminated gastric cancer. J Surg Res. 2013;185(1):119-26.

23. Kishi K, Yano M, Inoue M, Miyashiro I, Motoori M, Tanaka K, et al. Talaporfin-mediated photodynamic therapy for peritoneal metastasis of gastric cancer in an in vivo mouse model: drug distribution and efficacy studies. Int J Oncol. 2010;36(2):313-20.

24. Fidler IJ, Kripke ML. Metastasis results from preexisting variant cells within a malignant tumor. Science. 1977;197(4306):893-5.

25. Nowell PC. Mechanisms of tumor progression. Cancer Res. 1986;46(5):2203-7.

26. Shirasu N, Yamada H, Shibaguchi H, Kuroki M, Kuroki M. Potent and specific antitumor effect of CEA-targeted photoimmunotherapy. Int J Cancer. 2014;135(11):2697-710.

27. Wilson BC, Patterson MS. The physics, biophysics and technology of photodynamic therapy. Phys Med Biol. 2008;53(9):R61-109.

28. Ishida M, Kagawa S, Shimoyama K, Takehara K, Noma K, Tanabe $S$, et al. Trastuzumab-based photoimmunotherapy integrated with viral HER2 transduction inhibits peritoneally disseminated HER2negative cancer. Mol Cancer Ther. 2016;15(3):402-11.

29. Kepp O, Senovilla L, Vitale I, Vacchelli E, Adjemian S, Agostinis $\mathrm{P}$, et al. Consensus guidelines for the detection of immunogenic cell death. Oncoimmunology. 2014;3(9):e955691.

30. Mitsunaga M, Nakajima T, Sano K, Kramer-Marek G, Choyke $\mathrm{PL}$, Kobayashi $\mathrm{H}$. Immediate in vivo target-specific cancer cell death after near infrared photoimmunotherapy. BMC Cancer. 2012;12:345.

31. Sato K, Nagaya T, Choyke PL, Kobayashi H. Near infrared photoimmunotherapy in the treatment of pleural disseminated NSCLC: preclinical experience. Theranostics. 2015;5(7):698-709.

32. Sano K, Mitsunaga M, Nakajima T, Choyke PL, Kobayashi H. Acute cytotoxic effects of photoimmunotherapy assessed by $18 \mathrm{~F}-$ FDG PET. J Nucl Med. 2013;54(5):770-5.

33. Ogawa M, Tomita Y, Nakamura Y, Lee MJ, Lee S, Tomita S, et al. Immunogenic cancer cell death selectively induced by near infrared photoimmunotherapy initiates host tumor immunity. Oncotarget. 2017;8(6):10425-36. 